\title{
Research on the application of network public opinion in government crisis management
}

\author{
Li Zhigang ${ }^{1, a}$, Chen Hongqi ${ }^{2, b}$ \\ ${ }^{1}$ Jiangxi Science \& Technology Normal University, Nanchang, Jiangxi, 330013, P.R. China \\ ${ }^{\mathrm{a}}$ lizg98@126.com, ${ }^{\mathrm{b}}$ 375828970@qq.com
}

Keywords: The government, Crisis management, Internet public opinion, the Internet

\begin{abstract}
In today's society with the rapid development of network information, network public opinion development plays a more and more important role in crisis events, in the government crisis management also gradually learn to use the public opinion of network to carry on the control and treatment. But now the network public opinion in China there are lots of problems in the government crisis management, skill is not enough skilled, the relevant legal norms is not perfect and so on, so to explore and research the application of network public opinion in the government crisis management has strong practical significance.
\end{abstract}

\section{Introduction}

With the rapid development of Internet technology in China, its influence has penetrated into every aspect of our lives. Media people are free to express views, through the network public opinion, network public opinion more and arouse people's attention. Network public opinion is a double-edged sword, can open reduction events authenticity, widely mobilize the participation of the masses, also has certain one-sidedness and irrational, easy to induce the crisis, at the same time the network as the medium of the new forms of media has greatly increased the difficulty of government leading and control public opinion. This paper carefully studies the application of network public opinion in the government crisis management, at the same time, the better use put forward some constructive Suggestions, which to a certain extent, to maintain social order, guide the network public opinion to the positive, healthy and orderly development, is of great significance to achieve social harmony.

\section{The important role of the network of public opinion in the government crisis management}

Ensure rapid and full disclosure of information to meet the information needs of the public. Due to the occurrence of crises and uncertainty destructive, so once the crisis event occurs, the urgent need for the public to grasp the first time a comprehensive understanding of the real situation as much as possible the event, to avoid blind panic. Desire simultaneous crises to public events generated from the public reaction to events generated between there is a relatively short process, making the event a burst of public events often did not have time to make the right judgments, to obtain factual information on the impact of the facts own understanding. As the network of public opinion with the network media, to publish the first time crisis information and the information rich and comprehensive content, while the public media release timely event information, comprehensiveness, objectivity requirements make the network public opinion has been in a crisis event good play, to effectively meet the public anxious to know the whole incident, an urgent appeal to restore the truth. Compared with the traditional media, the network of public opinion timeliness and overall openness of this advantage is very beneficial to the government's information management crisis.

Dissemination efforts, affecting a wide range, improve social participation. Information dissemination network public opinion not only has the characteristics of speed, but also has spread intensity, affecting a wide range of features, any of the public are free to choose the information and the dissemination of information, the information can be both producers also disseminators of information, can to help people understand more extensive information on developments in the crisis 
sudden crisis in order to further broaden the spread and influence of crisis surface information for the development of crisis provides a good basis for information interaction and social solidarity. This information sharing behavior can lead to people's spirit of mutual help, get more social support and help to fully mobilize social participation of people.

Comprehensive variety of public information, strengthen executive power to resolve crises. Sudden outbreak of crises, often will be released at the beginning of a huge variety of information, and disseminate event information is usually with a preconceived characteristics, the first time released information is often able to guide people's perception of events and judgment. Therefore, in the event of crisis, the government can aid timely and comprehensive dissemination of the advantages of online media targeted event information to express their views on the crisis, public awareness will lead active, healthy side, rational and objective view of events development process. Meanwhile comprehensive variety of network public opinion to guide the healthy development of crises, mobilize social enthusiastic people, to strengthen executive power ending crises, earnestly safeguard the social stability.

\section{My Government is using the Internet to solve the crisis of public opinion during the Problems}

Network information sensitivity is low, relatively weak sense of crisis management. The rapid development of network information to enhance public understanding of the real situation of events, ability to obtain first-hand information, which also gives the government crisis management has brought new challenges. Although our government has recognized that the network management improved to some extent, but compared with the reality of crisis management level there is still a gap between network information sensitivity is low, relatively weak sense of crisis management. First in the lack of a certain crisis of information processing capability can not predict the rapid screening of crisis information network public opinion, information openness are not comprehensive, correct dissemination of information leading to crises there is a certain lag and delay, thus missing the development of the crisis Dynamic strangled in the cradle of a good opportunity. Secondly, there is no crisis management innovation, most of the relevant government departments to adopt a more traditional administrative means and methods to face and solve its network of public opinion crisis, just keep public opinion on the network blockade cut off, you want as much as possible to control public opinion, but often reach the desired effect, against the public's right to know while easily lead to social chaos appear.

Relevant laws and regulations are not sound enough, network management efficiency needs to be strengthened. Because of rapid development of information technology, its laws and regulations related to Internet governance does not keep pace, compared with most developed countries, the legal system is not perfect and mature. With the general increase in network applications and network technology, giving our life a lot easier at the same time, the network made Yao Pong vicious slander and casual events and other undesirable phenomena emerging, such behavior contrary to social morality seriously affecting people on crisis correct understanding and judgment of the event. However, China has yet to establish specific laws and regulations for effective management and sanctions for these events, not to be rigid network acting in legal penalties. Management behavior such education and technology combined with network management often leads to low efficiency, can not effectively prevent the emergence of a network of negative phenomena.

Network Information cohabitation, interference of public perception and judgment of events. Through the network platform, people can freely customize the publication and dissemination of the event view event information, not only to the true identity of the event spread, but also to conceal the identity of the event to express views and information dissemination. In this way not only can increase the amount of information of events, more information may appear spamming network defamation, false information swoop various negative phenomena, spread false news. It is very difficult to extract the cohabitation of mass information and select the correct and effective information on the crisis which affects cognition and judgment levels, causing social panic. 


\section{Strengthen the government using the network of public opinion crisis management advice and Measures}

First, the government must continue to broaden the channels of information disclosure, due to lower participation in the standard network public opinion body. Crisis events in the development process, the Government should make full use of various information dissemination way, take a variety of information dissemination, to strengthen openness and transparency crisis information. While the government should appropriate network media restrictions were relaxed newsgathering rights, freedom maximize the use of the characteristics of the network of public opinion, to enhance transparency of information dissemination event, make the network news media also have the power to edit and publish. Such a crisis event to make more rapid and objective truth can be presented to the public to help the government better improvement lags appeared previously reported delay phenomenon. Second, the government should continue to strengthen the network of laws and regulations related to further regulate Internet public opinion propagation behavior. Principle taken to analyze specific issues, the dissemination of information for the network of negative phenomena ,, formulate laws and regulations applicable operable to ensure that the behavior of individuals and units of the network specification, its effective supervision and guidance, thereby strengthening government In the crisis management process to control public opinion on the network. Finally, the government should take measures to establish the authority of the image of the body of mainstream public opinion, so as to better guide online public opinion towards a good direction. Governments can participate by inviting well-known experts and government leaders and other public discussion on the network, and establish social mainstream view, to strengthen the authority of the mainstream view, so that can be a good help the government manage the network of public opinion to guide the network of public opinion to the government desired The trend of development, maintain social stability.

\section{Conclusion}

All in all, the network of public opinion is the Information Age Government Crisis management requires greater attention and in-depth study of the subject, along with concern about the growing network events, network hot issues bound to lead to heated discussions majority of Internet users, the network of public crisis imminent, these all of the government crisis management, maintaining network stability and social order presents new challenges. Rulers should continue to strengthen the management of network public opinion, and earnestly safeguard citizens' rights, in the first period of crisis, the rapid development of appropriate treatment options, hide crisis, to reassure the public, to ensure a stable social order.

This paper is a research project of Humanities and social science research project in jiangxi province colleges and universities (NO. MKS1450).

\section{References}

[1] Y.H. Chen. On the Internet public opinion influence on the judiciary. Journal of the CPC Zhejiang Provincial Committee Party School, 2012 (2).

[2] L. Wang, D.P. Liu. Conduct policy and public opinion on the Internet Control Strategies. Journal of Jilin Public Security Academy, 2010 (1)

[3] Z.Y. Yi, Z.M. Ren. Major food safety accidents in media reports and interactive analysis of the characteristics of the event . Southeast propagation, 2011 (12) 